\title{
Transition from narrow to wide QRS complex tachycardia: What is the mechanism?
}

\section{Robert Pap MD, PhD iD | Cristina loana Tutuianu MD iD | László Sághy MD, PhD}

2nd Department of Medicine and Cardiology Centre, University of Szeged, Szeged, Hungary

Correspondence

Robert Pap, MD, PhD, 2nd Department of Medicine and Cardiology Centre, University of Szeged, Szeged H-6725, Hungary.

Email: pap.paprobert@gmail.com

A 50-year-old female presented with recurrent palpitations and documented wide QRS tachycardia. She underwent an electrophysiology study. During catheter manipulation, a narrow QRS tachycardia was induced (Figure 1). During attempted ventricular overdrive pacing, the narrow QRS tachycardia was transformed into a wide QRS complex tachycardia (Figure 1), which subsequently terminated spontaneously (Figure 2). What can be the mechanism of the two tachycardias?

The differential diagnosis of the narrow QRS tachycardia with a very short septal ventriculoatrial (VA) interval includes AV nodal reentry tachycardia (AVNRT) and atrial tachycardia (AT). During the wide, left bundle branch block (LBBB) morphology tachycardia, the His deflection is included in the ventricular electrogram on the Hisbundle recording, thereby excluding aberration and leaving ventricular tachycardia (VT) and pre-excited supraventricular tachycardia (SVT), that is, antidromic AV reentry or bystander pre-excitation, an option. The termination of wide QRS complex tachycardia without conduction to the atrium makes VT unlikely (Figure 2). SVT was further confirmed by an unchanged QRS morphology during atrial entrainment followed by an AVA postentrainment response. ${ }^{1}$ An atrial extrastimulus delivered from the high right atrium during a time when the AV node region was refractory advanced the next ventricular activation (Figure 3) proving the presence of an anterogradely conducting accessory pathway (AP) and pre-excited SVT. But the participation of the $A P$ in the tachycardia mechanism is not proven by this finding and bystander pre-excitation is still an option, especially in view of the narrow complex tachycardia.
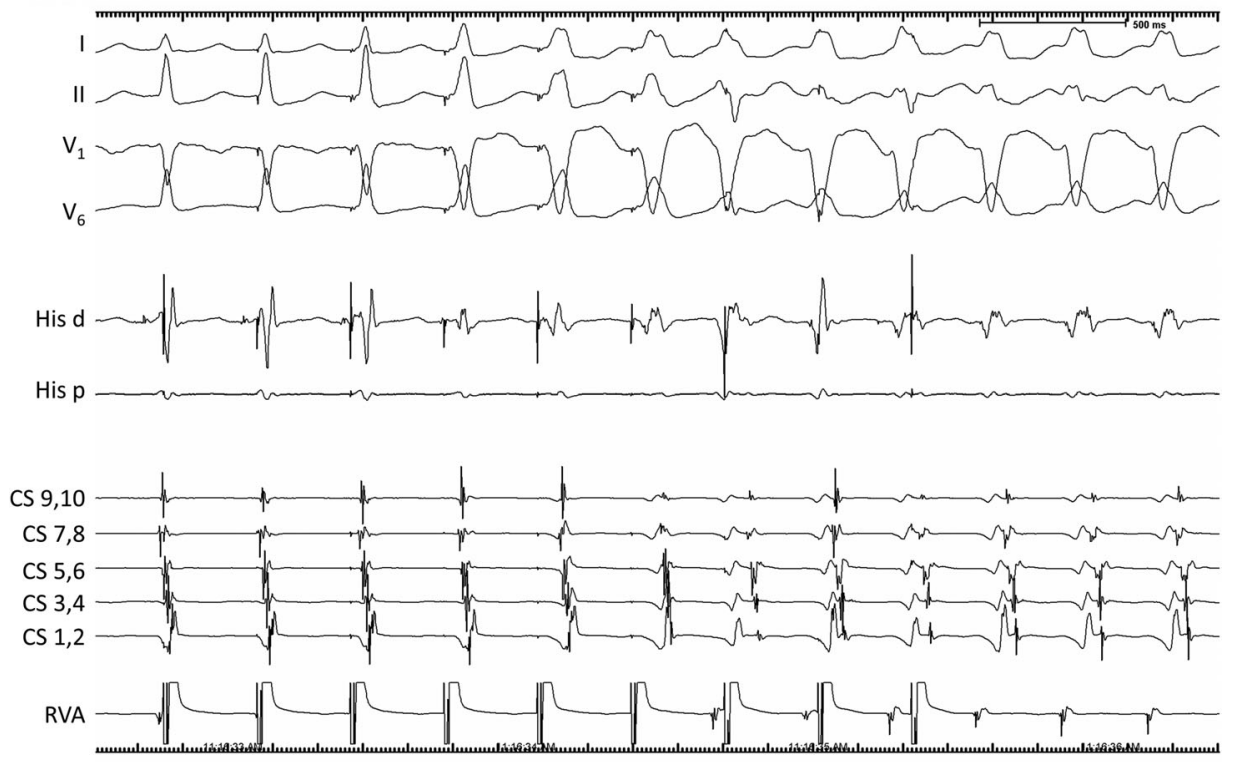

FIGURE 1 Surface ECG leads I, II, V1, and V6, together with intracardiac recordings from the His bundle region (His), coronary sinus (CS), and right ventricular apex (RVA) during attempted ventricular overdrive pacing transforming narrow QRS complex tachycardia to a wide complex tachycardia 

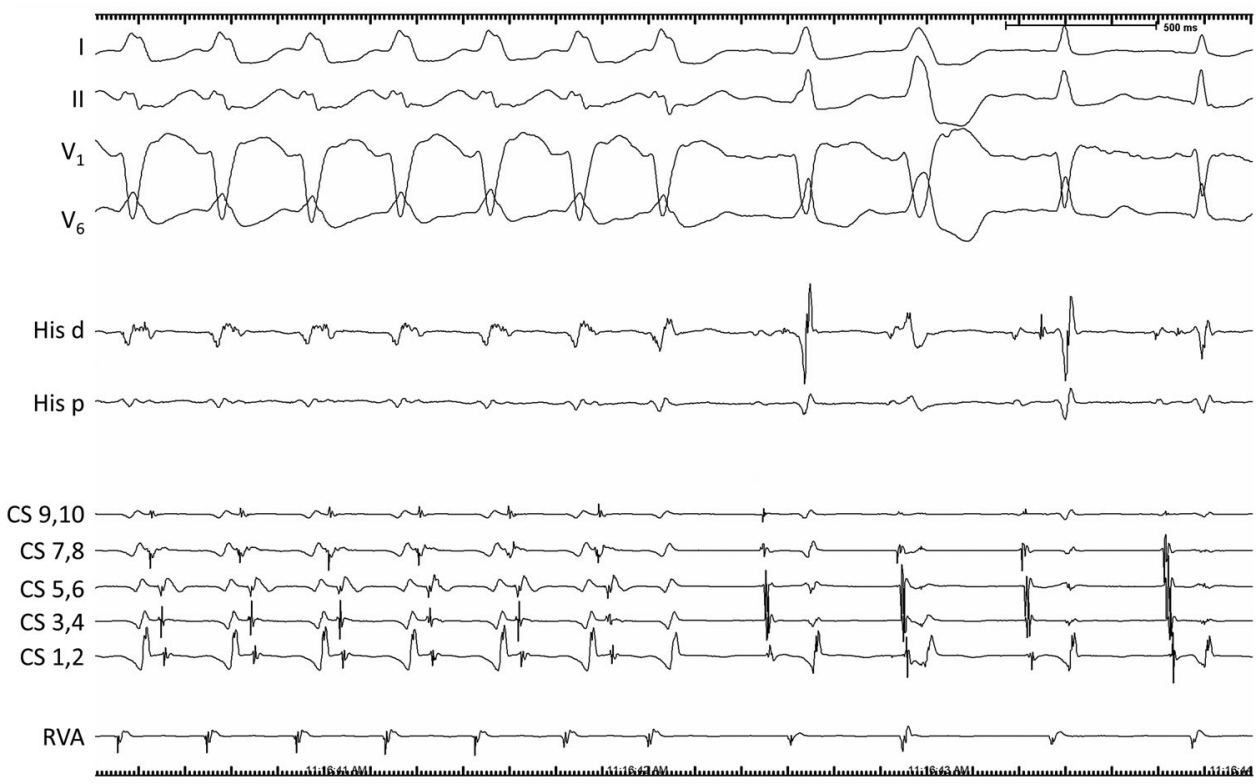

FIGURE 2 Spontaneous termination of wide complex tachycardia. Recording channels are the same as in Figure 1
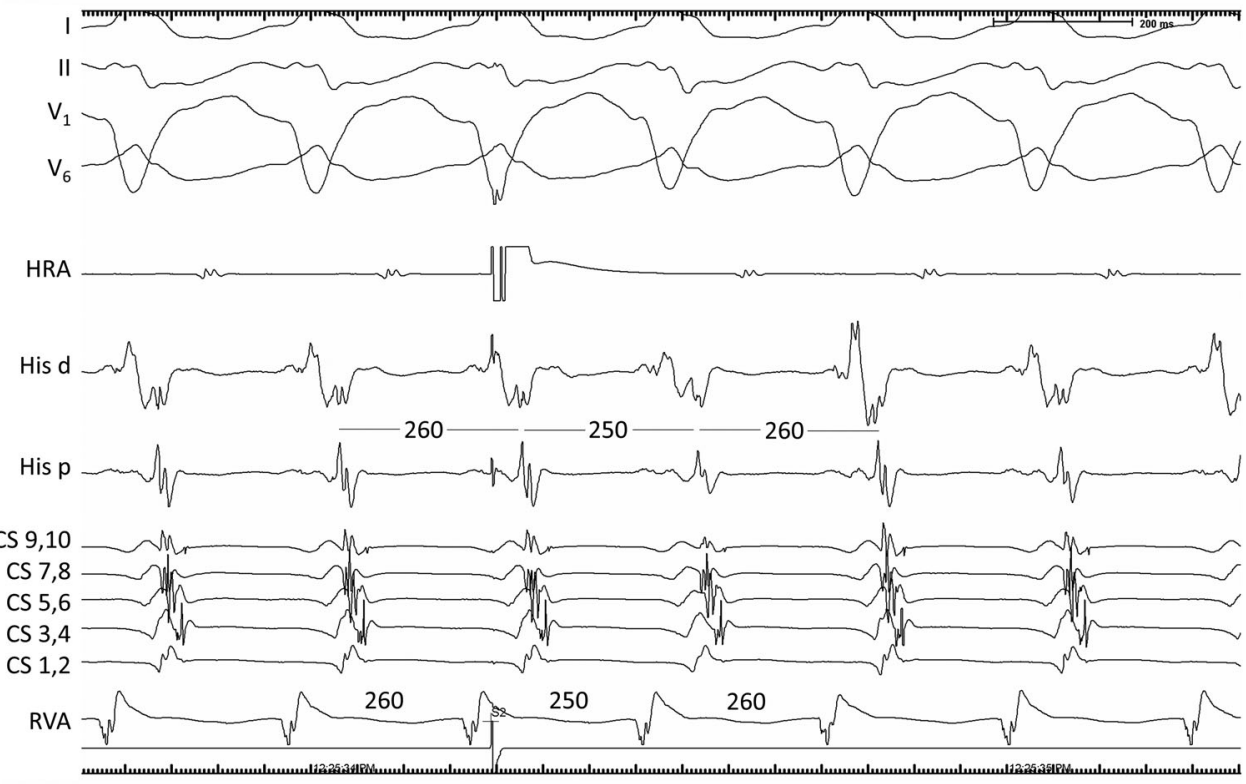

FIG URE 3 Premature atrial extrastimulus delivered from the high right atrium (HRA) during wide complex tachycardia. Other channels are the same as in Figure 1. Numbers are in milliseconds

It is noteworthy that after the atrial premature extrastimulus, not only is the next ventricular activation advanced but also the tachycardia is reset, indicated by the advancement of the subsequent atrial activation. Furthermore, the QRS morphology of the advanced beat is unchanged arguing against a fused QRS complex as with bystander pre-excitation. Therefore, the mechanism of the wide complex tachycardia was diagnosed as antidromic AV reentry.

A decremental, anterograde, atriofascicular (Mahaim) AP was mapped to the lateral tricuspid annulus and ablated successfully. Subsequently the narrow complex tachycardia was still inducible and ventricular entrainment excluded $\mathrm{AT}^{2}{ }^{2}$ Typical AVNRT was eliminated by slow pathway ablation. The patient remains free of symptoms during 6 years of follow-up.

\section{ORCID}

Robert Pap MD, PhD (iD https://orcid.org/0000-0002-7009-5063

Cristina loana Tutuianu MD (D)

https://orcid.org/0000-0003-0754-9584 


\section{REFERENCES}

1. Abdelwahab A, Gardner MJ, Basta MN, Parkash R, Khan A, Sapp JL. A technique for the rapid diagnosis of wide complex tachycardia with 1:1 $\mathrm{AV}$ relationship in the electrophysiology laboratory. Pacing Clin Electrophysiol. 2009;32(4):475-483.

2. Night BP, Zivin A, Souza J, et al. A technique for the rapid diagnosis of atrial tachycardia in the electrophysiology laboratory. J Am Coll Cardiol. 1999;33(3):775-781.
How to cite this article: Pap R, Tutuianu $\mathrm{Cl}$, Sághy $\mathrm{L}$. Transition from narrow to wide QRS complex tachycardia: What is the mechanism? Pacing Clin Electrophysiol. 2020;1-3. https://doi.org/10.1111/pace.13877 